\title{
Carotid Artery Stenting With Filter Protection in High- Risk Patients Showing Severe Electroencephalographic Alterations During Carotid Endarterectomy
}

\author{
Roberto Gandini, MD1; Alessio Spinelli, MD1; Sebastiano Fabiano, MD1; \\ Vittorio Colangelo, MD; Maria Grazia Marciani, MD, PhD²; Andrea Romigi, MD²; and \\ Giovanni Simonetti, MD, PhD ${ }^{1}$
}

Departments of ${ }^{1}$ Diagnostic Imaging and Interventional Radiology and ${ }^{2}$ Neuroscience, University of Tor Vergata, Rome, Italy.

\begin{abstract}
$\checkmark$
Purpose: To describe the results and efficacy of stent treatment in patients with carotid stenosis who had aborted carotid endarterectomy procedures due to the appearance of severe electroencephalographic (EEG) alterations.

Methods: A retrospective study was conducted of 18 patients (11 men; mean age 72 years, range $62-84)$ with symptomatic high-grade carotid artery stenoses ( $\geq 70 \%$ ) who experienced severe EEG alterations during carotid endarterectomy, causing the procedure to be aborted. Twelve patients had shown no hemodynamic alterations during preoperative transcranial Doppler evaluation after external compression of the common carotid artery; the remaining 6 could not be evaluated. The patients were referred for carotid artery stenting (CAS); 7 had contralateral internal carotid artery stenosis and 5 had contralateral occlusion. Endovascular intervention was carried out using standard techniques under filter protection. Follow-up was scheduled at 3, 6, and 12 months.

Results: All patients were successfully treated without immediate complications. The EEG did not display any significant alterations during the endovascular procedure. Mean followup was 43 months. Magnetic resonance imaging at 6 months showed no signs of cerebral ischemia. Color Doppler ultrasound imaging documented normal stent patency in all patients.

Conclusion: Patients with symptomatic severe carotid stenosis ( $\geq 70 \%$ ) who are considered at risk due to the appearance of severe EEG alterations during surgical treatment may benefit from CAS with respect to both major and minor complications. Larger studies are needed to confirm these findings.
\end{abstract}

J Endovasc Ther 2006;13:451-456

Key words: carotid endarterectomy, carotid artery, stenosis, electroencephalography, cerebral ischemia, carotid angioplasty, stent

Endovascular treatment of carotid stenosis is a valid alternative to carotid endarterectomy (CEA) owing to improved devices and techniques and a growing number of studies showing that carotid artery stenting (CAS) with cerebral protection is not inferior to CEA in terms of major and minor postprocedural complications. ${ }^{1-4}$

The role of the endovascular technique is particularly important in high-risk patients in whom the surgical approach may be dangerous due to inadequate cerebral collateraliza-

The authors have no commercial, proprietary, or financial interest in any products or companies described in this article.

Address for correspondence and reprints: Vittorio Colangelo, MD, Viale Oxford 81, 00133 Rome, Italy. Fax: 39-06-20902404; E-mail: vcolan@tiscali.it 
tion. Furthermore, concomitant contralateral stenosis or occlusion could pose a greater risk of neurological complications due to abrupt reduction in blood flow after clamping of the carotid vessels. In this respect, the North American Symptomatic Carotid Endarterectomy Trial (NASCET) showed an increase in perioperative risk after endarterectomy in patients with contralateral internal carotid artery (ICA) occlusion ${ }^{5}$; but it is uncertain whether a contralateral occlusion could cause a periprocedural stroke during CEA. ${ }^{6,7}$ For CAS, however, a few authors have found that endovascular treatment in the setting of bilateral disease is not encumbered by this periprocedural risk. ${ }^{8-10}$

Electroencephalography (EEG) is one of the most commonly used methods of monitoring possible cerebrovascular insufficiency during CEA. ${ }^{11}$ Variations in EEG activity may be due to surgery (i.e., clamping) or nonsurgical causes, such as anesthetic level, blood pressure, other physiological factors. ${ }^{12}$ Specific signs of altered electrical activity during surgery may induce the surgeon to abort the procedure, especially when such alterations appear early and in patients with comorbidities. Patients afflicted with atherosclerotic disease usually have involvement in multiple vascular territories, so, apart from the possible coronary comorbidities, the vascular autoregulatory system may be altered, thus affecting the redistribution of flow and cerebral perfusion during the procedure.

Our study evaluated the results and efficacy of angioplasty and carotid stenting with cerebral protection in patients in whom CEA had been aborted because of the appearance of serious EEG alterations during carotid clamping.

\section{METHODS}

\section{Patient Characteristics}

From December 1998 to May 2004, the endovascular team of our department treated 331 patients for occlusive disease in 363 ICAs. Eighteen of these patients (11 men; mean age 72 years, range $62-84$ ) with a symptomatic de novo ICA stenosis $>70 \%$ were referred for CAS after surgical endarterectomy was abort- ed due to serious EEG alterations during carotid clamping. Comorbidities included diabetes $(n=13)$, hypertension $(n=13)$, prior stroke $(n=3)$, hypercholesterolemia $(n=10)$, and obesity $(n=5)$.

Twelve (67\%) of the 18 patients had had a preoperative transcranial Doppler (TCD) scan performed while compressing the common carotid artery (CCA) to simulate clamping. Seven patients showed no significant hemodynamic alterations in the contralateral middle cerebral artery. The other 5 patients, all with contralateral ICA occlusion, showed mild hemodynamic alterations without significant blood flow reduction, so the surgical team planned to use an intraoperative shunt during CEA in these patients. The remaining 6 patients lacked a suitable acoustic window for $T C D$, but none of them had contraindications to surgery and so underwent CEA.

Within the first 20 seconds after clamping, each patient manifested serious EEG alterations (Fig. 1), either a decrease in amplitude of all activity, including delta and faster frequencies, or increased amplitude and duration of delta waves. Our surgeons considered the precocious appearance of major EEG alterations after clamping as a possible threat to the positioning of a carotid-carotid shunt, even though it can be performed in $\sim 60 \mathrm{sec}-$ onds. Considering the patients' comorbidities, the surgeons did not feel that they could position a shunt safely, so they aborted the procedures.

\section{Carotid Stent Procedure}

When the patients were referred for endovascular treatment, color Doppler sonographic analysis of plaque morphology documented hyperechogenicity in 9 cases, mixed morphology in 7 cases, and hypoechogenicity in 2, one of which showed an ulcerated plaque. Computed tomography (CT) and/or magnetic resonance angiography (MRA) confirmed the significant stenosis and provided further information on the circle of Willis and the anatomy of the supra-aortic vessels. Seven patients had contralateral ICA stenoses (4 $>70 \%$ and 3 between $50 \%$ and $60 \%$ ), and 5 had contralateral occlusions (2 CCA, 3 ICA); 


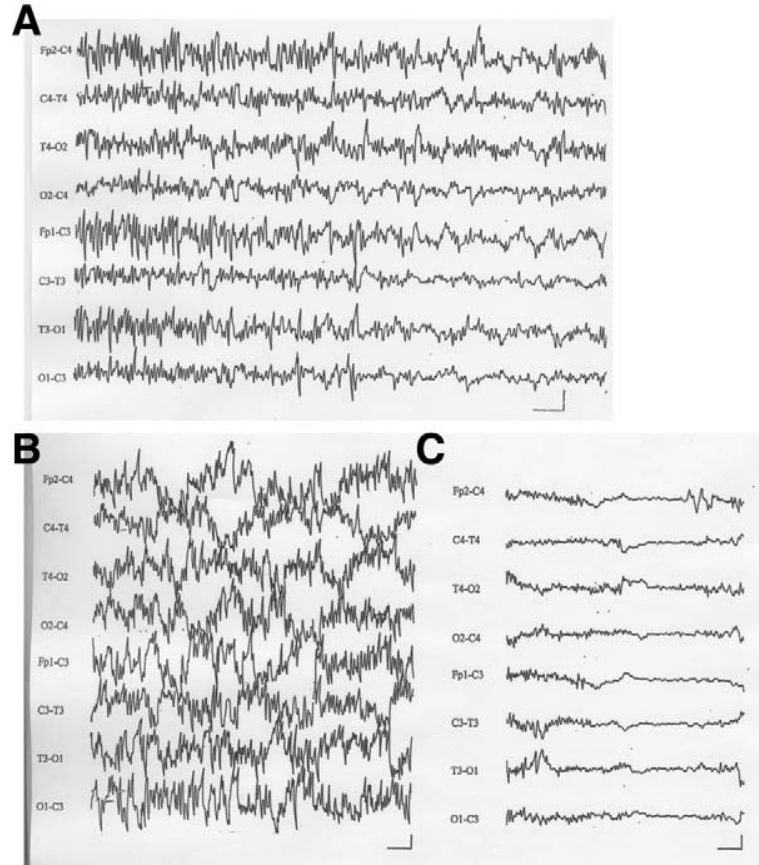

Figure $1 \bullet$ (A) In this patient with left carotid artery stenosis, carotid clamping induced an increase in amplitude of slow waves (1- $\mathrm{Hz}$ frequency) and simultaneous reduction in faster frequencies localized in the left front, central, and temporal regions. (B) In another patient with right carotid stenosis and contralateral carotid occlusion, the EEG before clamping showed widespread fast frequencies with high voltage. (C) Right carotid clamping induced severe, bilateral reduction in amplitude of faster frequency components. EEGs were recorded with a $0.3-\mathrm{Hz}$ high band-pass filter and a $50-\mathrm{Hz}$ low band-pass filter. The horizontal bars indicate $1 \mathrm{sec}-$ ond; the vertical bars are $20 \mu \mathrm{V}$.

the other 6 had no significant disease in the contralateral carotid circulation.

The patients were pretreated with $100 \mathrm{mg} / \mathrm{d}$ of aspirin and $75 \mathrm{mg} / \mathrm{d}$ of clopidogrel for at least 4 to 5 days prior to treatment. During CAS, continuous EEG monitoring was performed using an analogue 10-channel system polygraph (Vega 10; EBNeuro, Florence, Italy). The time constant was 0.3 seconds, and a 50$\mathrm{Hz}$ low-pass filter was needed in the operating room's electrical environment. Electrodes were positioned according to the 10-20 International System to monitor 8 EEG channels (Fp2, C4, T4, O2, Fp1, C3, P3, O1). The recording was begun before the procedure and continued throughout, with careful attention to the EEG during the following critical phases: guide catheter manipulation in the CCA, advancement of the catheter or long introducer into the CCA, guidewire manipulation through the stenosis, predilation, crossing the stenosis with the protection filter, stent advancement and release, post dilation, and filter recovery.

During the procedure, heparin boluses (50$100 \mathrm{U} / \mathrm{kg}$ ) were administered to maintain the activated coagulation time around $300 \mathrm{sec}-$ onds. All the procedures were performed with continuous heparinized saline wash to prevent the formation of clots or air emboli in the system. In 7 cases, the "over-the-wire" technique was used, in which the ipsilateral external carotid artery was catheterized from a femoral access with a 0.035 -inch hydrophilic guidewire and diagnostic catheters. After exchanging for a stiff or Amplatz guidewire, a long 6-F introducer (Cook Inc., Bloomington, IN, USA) was advanced in the CCA. In 9 cases, catheterization of the CCA was done with a 0.035 -inch stiff hydrophilic guidewire and 7-F guiding catheter. Owing to pronounced tortuosity of the supra-aortic vessels (type III arch) in 2 patients, a coaxial telescopic system was used, in which a $5-\mathrm{F}(125-\mathrm{cm})$ diagnostic catheter and 0.035 -inch stiff guidewire were inserted inside a catheter or a long introducer and advanced simultaneously. An EPI FilterWire cerebral protection filter (Boston Scientific, Natick, MA, USA) was deployed in all patients. Predilation with a 3-mm balloon was necessary in only 1 case because of a tight stenosis. Monorail Carotid Wallstents (Boston Scientific) were used exclusively. Atropine $(0.5-1.0 \mathrm{mg})$ was administered before stent dilation, which was done with 5- to 6$\mathrm{mm}$ balloons (Fig. 2) for an average of 3 to 7 seconds.

Postoperatively, clopidogrel was continued for 6 weeks and aspirin indefinitely. Follow-up consisted of clinical examination with color Doppler imaging before discharge and at 3,6, and 12 months, with semiannual examinations thereafter.

\section{RESULTS}

In all patients, the filter was correctly placed distal to the stenosis with no technical prob- 


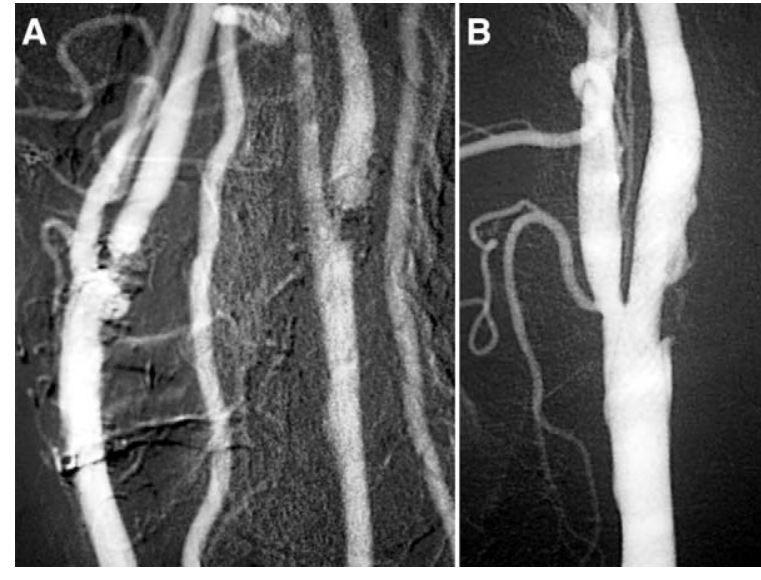

Figure $2 \diamond(A)$ This DSA shows evidence of significant ostial stenosis of both left and right ICAs. (B) Postprocedural DSA after stent deployment in the left ICA.

lems. Positioning the stent was successful in all cases, which lasted an average of $23 \mathrm{~min}$ utes (range 12-47) from the femoral artery puncture to sheath removal. In 4 patients, macroscopic debris was found inside the filter.

Continuous intraoperative EEG monitoring (Fig. 3) did not show significant cerebral alterations during any phase of the procedure. The final angiogram showed good stent position with restoration of the physiological vessel lumen (residual stenosis $<20 \%$ ) and no changes in the circle of Willis.

Over a mean 43-month follow-up (range 12-65), no patient showed either clinical evidence of neurological deficits at the 3 and 6month examinations or MR signs of cerebral ischemia at 6 months. All stents were patent at the 12-month follow-up.

\section{DISCUSSION}

With recent developments in cerebral protection systems, miniaturization of devices, and evolutionary stent materials, CAS has become a safer technique, with a low risk of complications. ${ }^{1-4}$ The Pro-CAS registry of 3267 procedures confirmed the technical success of CAS $(98 \%)$, with $0.6 \%$ mortality and $1.2 \%$ major and $1.3 \%$ minor stroke rates. ${ }^{4}$ The combined stroke and death rate was $2.8 \%$. The randomized SAPPHIRE trial compared the

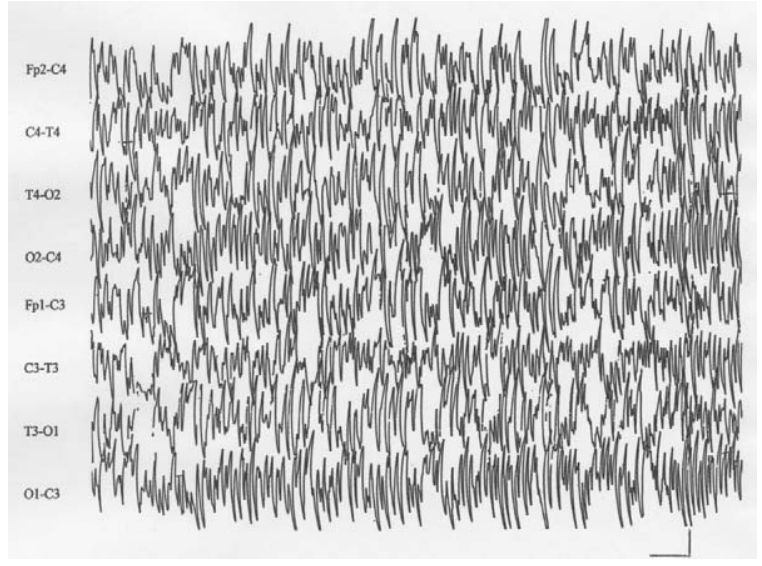

Figure 3 An EEG showing no significant changes in amplitude or frequency during each phase of the endovascular procedure $(0.3-\mathrm{Hz}$ high band-pass filter, $50-\mathrm{Hz}$ low band-pass filter). The horizontal bar indicates 1 second; the vertical bar is $20 \mu \mathrm{V}$.

results of CEA (151 patients) to those of CAS under cerebral protection (156 patients) in symptomatic patients with $\geq 50 \%$ stenosis or asymptomatic patients with $\geq 80 \%$ stenosis. $^{3}$ Notably, several CAS patients had been excluded from the surgical protocol because of at least 1 risk factor, such as a high degree of heart failure, severe pulmonary disease, contralateral carotid occlusion, previous surgery with restenosis, or previous neck radiotherapy. The study clearly demonstrated a lower rate of complications (i.e., mortality, periprocedural neurological events, and myocardial infarction) at 30 days in the CAS patients, supporting the validity of protected CAS as an option in the treatment of carotid stenoses.

In our patients, the use of a cerebral protection system seems to have played a fundamental role in allowing continuous antegrade flow in the target carotid axis, thus preserving cerebral perfusion. During CEA, on the other hand, the abrupt reduction in cerebral blood flow due to carotid clamping in patients with contralateral ICA or CCA occlusion may increase the risks of the procedure. The NASCET study reported a $14 \%$ increased risk of cerebral ischemia in patients with contralateral ICA occlusion, directly related to a decrease in cerebral blood flow due to bilateral carotid pathology. ${ }^{5}$ In a systematic review of 1729 patients from the European Carotid Sur- 
gery Trial, Rothwell et al. ${ }^{13}$ described a 2 -fold risk of perioperative stroke in patients with contralateral carotid occlusion. On the other hand, Samson et al. ${ }^{14}$ did not find any significant change in surgical perioperative risk for patients with significant occlusions or stenosis of the contralateral ICA. Nevertheless, we believe that the presence of a contralateral occlusion or significant stenosis can be a risk factor during endarterectomy, particularly in high-risk patients with diffuse atherosclerosis and comorbidities.

In a study by Samson et al., ${ }^{14} 2$ major types of clamp-related EEG variations were detailed: attenuation of all activity by at least $75 \%$ and/or a $\geq 2$-fold increase in $\leq 1-\mathrm{Hz}$ delta activity, which is what occurred in our study patients. In the absence of a contralateral carotid occlusion or severe stenosis, EEG changes with clamping are usually ipsilateral. Bilateral clamp-related changes typically arise when contralateral blood flow is severely compromised. The incidence of unilateral changes is more than twice that of bilateral changes. ${ }^{12}$ The incidence of the major variations noted above range from $3.4 \%$ to $12.5 \%{ }^{15}$ owing to several variables, such as subjective elements in assessing non-processed EEG signals, different high band-pass filter frequencies, varying anesthetic techniques, and different patient populations.

Since diffuse atherosclerosis in the entire arterial district can alter flow regulatory systems mediated by intraparietal receptors, patients without significant stenosis or occlusion of the contralateral carotid axis can nonetheless be considered at high surgical risk. Moreover, the absence of communicating arteries may explain the intolerance to clamping of the contralateral carotid. In 2 of our 18 cases, the DSA performed before the stent procedure confirmed an anatomical variation characterized by the absence of the anterior and posterior communicating arteries. In the remaining 16 cases, no anatomical variations of the intracranial vessels were detected.

Some authors report that TCD is an excellent means of predicting cerebral ischemia during carotid surgery. ${ }^{16}$ Consistent with the work by Lagneau et al., ${ }^{17}$ our study showed that the preliminary evaluation with TCD does not present a reliable prediction of hemodynamic compensation in the contralateral carotid territory. In addition, the frequent absence of an acoustic window for TCD and the difficulty of external compression to simulate clamping in patients with widespread parietal calcification reduce the potential value of TCD. In our group, 7 patients who did not show hemodynamic alterations in the preliminary TCD evaluation demonstrated severe EEG alterations within the first 20 seconds after clamping, which suggests widespread atherosclerotic pathology with abnormal adaptation of flow redistribution in the intracranial vessels. This hypothesis may explain the severe EEG alterations in those patients with regular patency and caliber of the contralateral carotid artery.

\section{Conclusion}

In our experience, stent placement with a protection system may represent the treatment of choice in patients with high surgical risk because of stenosis or occlusions of the contralateral carotid artery or because of serious, widespread atherosclerotic pathology that involves the supra-aortic vessels. Furthermore, the application of carotid stenting in high-risk patients suggests that endovascular management of carotid stenoses can be a valid alternative to CEA, achieving high technical success and a low rate of complications.

\section{REFERENCES}

1. CARESS Steering Committee. Carotid Revascularization using Endarterectomy or Stenting Systems (CARESS): phase I clinical trial. J Endovasc Ther. 2003;10:1021-1030.

2. Goodney PP, Schermerhorn ML, Powell RJ. Current status of carotid artery stenting. J Vasc Surg. 2006;43:406-411.

3. Yadav JS, Wholey MH, Kuntz RE, et al. Protected carotid-artery stenting versus endarterectomy in high-risk patients. $N$ Engl $J$ Med. 2004;351:1493-1501.

4. Theiss W, Hermanek P, Mathias K, et al. ProCAS. A prospective registry of carotid angioplasty and stenting. Stroke. 2004;35:2134-2139.

5. Ferguson GG, Eliasziw M, Barr HW, et al. The North American Symptomatic Carotid Endar- 
terectomy Trial: surgical results in 1415 patients. Stroke. 1999;30:1751-1758.

6. Mozes G. High-risk carotid endarterectomy. Semin Vasc Surg. 2005;18:61-68.

7. Grego F, Antonello M, Lepidi S, et al. Is contralateral carotid artery occlusion a risk factor for carotid endarterectomy? Ann Vasc Surg. 2005; 19:882-889.

8. Mathur A, Roubin GS, Gomez CR, et al. Elective carotid artery stenting in the presence of contralateral occlusion. Am J Cardiol. 1998;81: 1315-1317.

9. Mericle RA, Kim SH, Lanzino G, et al. Carotid artery angioplasty and use of stents in high-risk patients with contralateral occlusions. J Neurosurg. 1999;90:1031-1036.

10. Sabeti S, Schillinger M, Mlekusch W, et al. Contralateral high grade carotid artery stenosis or occlusion is not associated with an increased risk for poor neurological outcome after carotid stenting. Radiology. 2004;230:70-76.

11. Findlay JM, Marchak BE, Pelz DM, et al. Carotid endarterectomy: a review. Can J Neurol Sci. 2004;31:22-36.

12. Blume WT, Ferguson GG, McNeill DK. Signifi- cance of EEG changes at carotid endarterectomy. Stroke. 1986;17:891-897.

13. Rothwell PM, Slattery J, Warlow CP. Clinical and angiographic predictors of stroke and death from carotid endarterectomy: systematic review. BMJ. 1997;315:1571-1577.

14. Samson RH, Showalter DP, Yunis JP. Routine carotid endarterectomy without a shunt, even in the presence of a contralateral occlusion. Cardiovasc Surg. 1998;6:475-484.

15. Sundt TM, Sharbrough FW, Piepgras DG, et al. Correlation of cerebral blood flow and electroencephalographic changes during carotid endarterectomy: with results of surgery and hemodynamics of cerebral ischemia. Mayo Clin Proc. 1981;56:533-543.

16. McDowell HA, Gross GM, Halsey JH. Carotid endarterectomy monitored with transcranial Doppler. Ann Surg. 1992;215:514-518.

17. Lagneau $P$, Baujat $B$, Anidjar $S$, et al. Is transcranial Doppler a worthwhile examination for preoperative evaluation of the circle of Willis? Evaluation of 137 carotid endarterectomies performed under regional anesthesia. Int Angiol. 1998;17:168-170. 